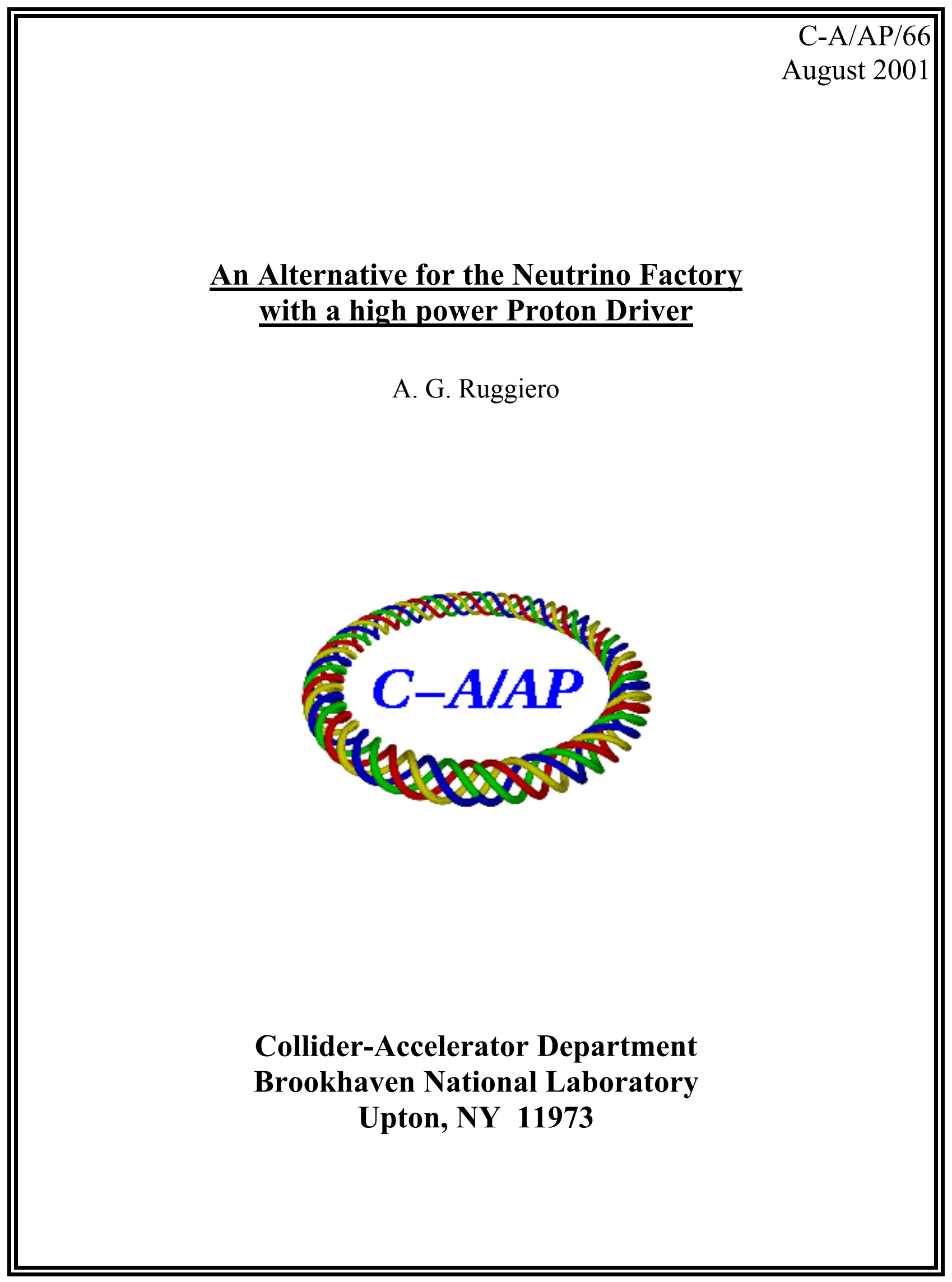




\title{
An Alternative Scheme for the Neutrino Factory with a High Power Proton Driver
}

\author{
Alessandro G. Ruggiero \\ Brookhaven National Laboratory \\ August 1, 2001
}

\begin{abstract}
We describe a scheme to produce an intense and collimated beam of neutrinos for the neutrino-oscillation experiment. The scheme feature is the presence of a Proton Driver that generates a proton beam at very large power $(10 \mathrm{~mA} \times 15 \mathrm{GeV})$, considerably higher than that proposed elsewhere for this application. With this scheme, because of the high intensity of the proton beam, to produce neutrinos at the same required rates, it is sufficient to collect $\pi$ and $\mu$ mesons only around a small angle and at reduced momentum spreads. This eliminates the need for the difficult longitudinal manipulations of the protons and mesons, and of the ionization cooling that still needs to be demonstrated. It is also shown, at the end of the paper, that the Neutrino Factory here proposed can also be used as an injector for a $1 \mathrm{x} 1 \mathrm{TeV}^{2} \mu^{+}-\mu^{-}$collider at large luminosity.
\end{abstract}

\section{Introduction}

A Collaboration team, composed of several institutions, has put forward a proposal [1] for a Neutrino Factory that should be located at Brookhaven National Laboratory. The intent is to aim the Neutrino Beam at some location in New Mexico. The production rate of the neutrinos is about $2 \times 10^{14} / \mathrm{s}$, centered around the use of a Proton Driver with an average power of $1 \mathrm{MW}$ [2]. Because of the relatively low intensity of the primary proton beam, and the fact that the beam from the AGS is bunched at low frequency, to arrive at the prefixed production rate, complicate proton bunch manipulation, that includes stacking and compression, are required. Also the operation of the proton source is pulsed with a low duty cycle that causes difficulties to the stability of the target, that has then be taken to be liquid mercury. To accept as many meson particles as possible, large angular and longitudinal acceptance of the secondary particles are required with complicate production and transport channel. Also the secondary beams need to be bunch-rotated before final acceleration. The large transverse spread is problematic and one needs to resort to some cooling, like ionization cooling, that though still needs to be demonstrated.

To avoid most of these problems, the scheme proposed in this report makes use of a novel concept of Proton Driver that generates a continuous proton beam at much higher power (150 MW versus $1 \mathrm{MW}$ of the Collaboration Proposal). Moreover, the protons are accelerated in a sequence of Super-Conducting Linacs, and the beam in exit is bunched at high frequency. The proton bunches have then small dimensions and spreads. RF manipulation of the proton bunches is not required, and target stability problems are not a concern. At production and during transport, the $\pi$ and $\mu$ mesons have the same high frequency bunching of the proton beam. Considering the relatively small dimensions also 
of the $\mu$-beam, this is directly transferred and accelerated in a sequence of SuperConducting Linacs to $32 \mathrm{GeV}$. Ionization cooling to reduce the beam emittance and momentum spread is then not needed. Also not needed is further bunch compression and rotation of the $\mu$-bunches.

The proposed Neutrino Factory can also be used as an injector to a $1 \times 1-\mathrm{TeV}^{2} \mu^{+}-\mu^{-}$ collider. After acceleration to $32 \mathrm{GeV}$, the muons of both signs are accelerated further in a more efficient Super-Conducting Linac to $1 \mathrm{TeV}$. The mesons with opposite sign are separated and transferred into a common storage ring where they circulate with a lifetime of $20 \mathrm{~ms}$. Multi-turn stacking of the bunches is done only with "momentum painting" to preserve the initial small emittance, and to reduce the number of circulating bunches. A luminosity of about $1 \times 10^{31} \mathrm{~cm}^{-2} \mathrm{~s}^{-1}$ is expected.

\section{Neutrino Production}

The neutrino production follows the following chain of reactions and decays:

$$
\mathrm{p}+\mathrm{X} \quad \rightarrow \pi^{ \pm} \quad->\mu^{ \pm} \quad->v
$$

As shown in Figure 1, a proton of momentum $\mathrm{p}_{0}$ impinges on a heavy nucleus $\mathrm{X}$. From the scattering, a $\pi^{ \pm}$meson (mass at rest $\mathrm{m}_{\pi}=139.5675 \mathrm{MeV}$ ) is produced with momentum $\mathrm{p}_{\pi}$ and at an angle $\theta_{\pi}$ from the direction of incidence. The meson decays with a lifetime (at rest) of $2.603 \times 10^{-8} \mathrm{~s}$ into a neutrino $v$ and a $\mu^{ \pm}$meson. In turn the $\mu^{ \pm}$meson (mass at rest $\mathrm{m}_{\mu}=105.6595 \mathrm{MeV}$ ), that was produced with momentum $\mathrm{p}_{\mu}$, decays in $\mathrm{e}^{ \pm}+v_{\mu}+\bar{v}_{\mathrm{e}}$ with a lifetime (at rest) of $2.1971 \times 10^{-6} \mathrm{~s}$.

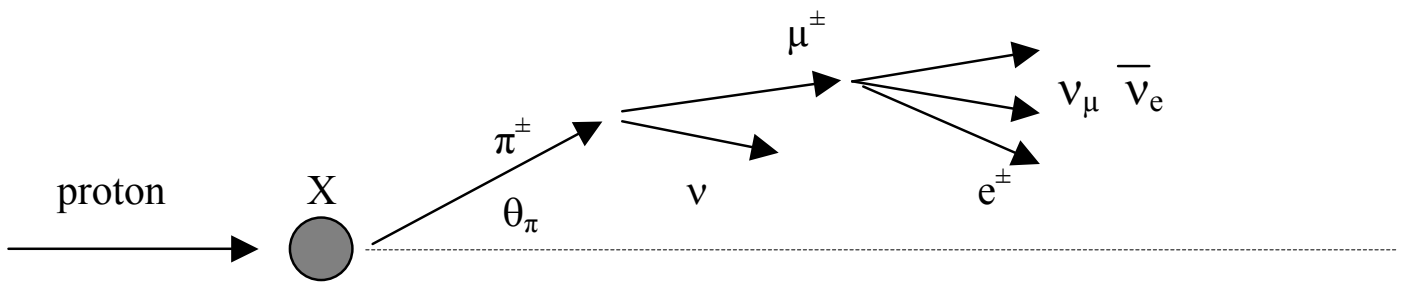

Figure 1. Chain of reactions and decays for the neutrino production

For the neutrino-oscillation experiment, used to determine the neutrino mass with high precision, the neutrinos are required to travel a long distance of several thousand of kilometers. It is then important for this experiment that the neutrino beam is produced well collimated to avoid too much angular dispersion at the arrival point. Because the $\pi^{ \pm}$ mesons are produced with a large transverse momentum spread, the neutrinos from their decay are thus not very useful. Moreover, the $\pi^{ \pm}$mesons have a too short lifetime because one can act on them to reduce their angular spread before they decay. On the other side the lifetime of the $\mu^{ \pm}$mesons, if their energy is large enough, is sufficiently long to operate on them to reduce their transverse spread simply by acceleration. Conversely 
then, it is hoped that the two types of neutrino from the $\mu^{ \pm}$decay form a beam collimated enough to be useful at the long required distance.

The assumption made here is that of course the neutrino mass, if any, is very small and in principle negligible for the estimate of the kinematics of the reactions and decays of Figure 1 where scattering angles have been exaggerated. The angular distribution and momentum spectrum of the $\pi^{ \pm}$mesons [3] are shown in Figures 2 and 3. We shall assume, for simplicity, that the $\mu^{ \pm}$mesons will have also about the same distribution and that they follow closely the "footprint" of the $\pi^{ \pm}$- meson beam.

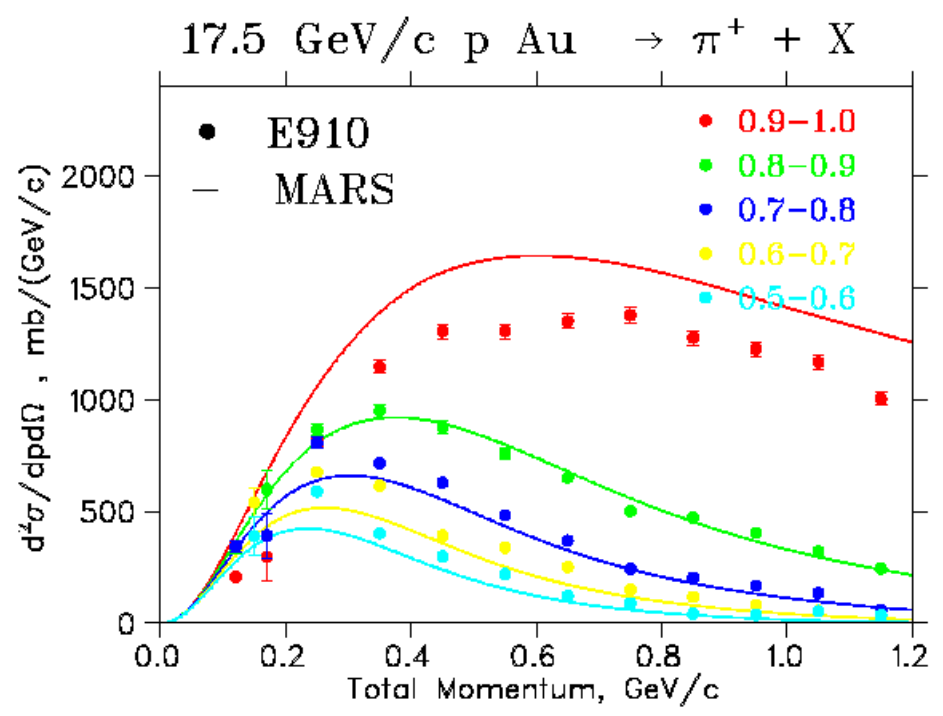

Figure 2. Angular and momentum distribution of the $\pi^{+}$-meson production

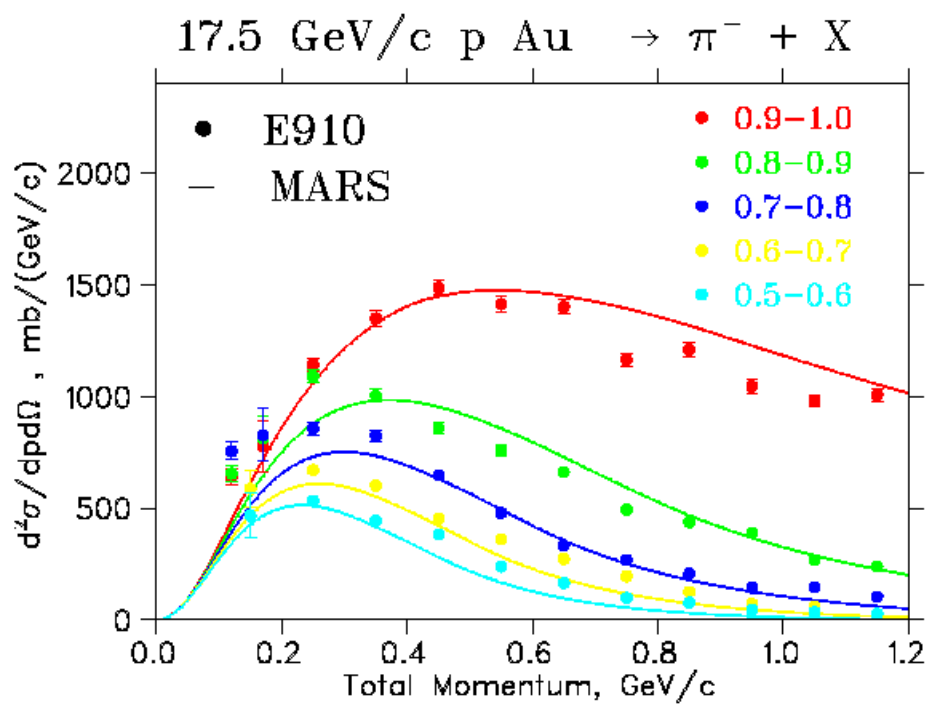

Figure 3. Angular and momentum distribution of the $\pi^{-}$-meson production 


\section{An alternative Scenario based on a High-Power Proton Driver}

The scenario we are here proposing for the Neutrino Factory is sketched in Figure 4. It is made of four parts:

(1) a $15-\mathrm{GeV}$ Proton Driver (PD),

(2) a $\pi-\mu$ Production Channel $(\pi \mu \mathrm{PC})$, that is a solid target immediately followed by a transport channel made of a super-conducting 20-T solenoid magnet where the $\pi$ mesons decay and the $\mu$ mesons are produced,

(3) an accelerating section consisting of a $2-\mathrm{GeV} \mathrm{SCL}$ with two re-circulating SCLs ( $\mu \mathrm{SCL}$ ) for the acceleration of the $\mu$ mesons to $32 \mathrm{GeV}$, and

(4) a 32-GeV muon Storage Ring ( $\mu \mathrm{SR})$, where the $\mu$ mesons circulate until they decay in neutrinos.

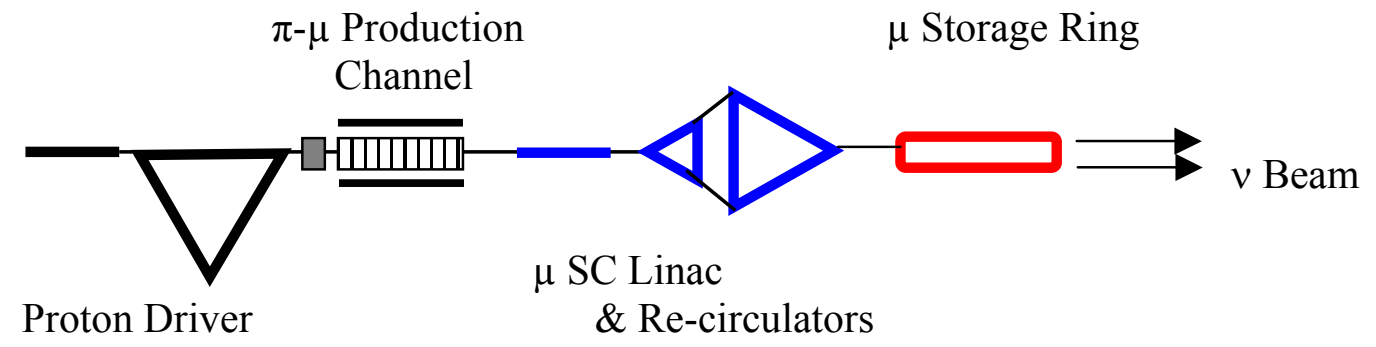

Figure 4. Neutrino Factory

No ionization cooling, nor bunch rotation and/or compression of the primary proton beam and of the $\mu$-beam are here required. The main feature of the proposed scheme is the high power of the Proton Beam.

The rate of $\mu$-production $\mathrm{dn} / \mathrm{dt}$ is in linear approximation proportional to the proton beam intensity $\mathrm{I}_{\mathrm{p}}$, to the momentum bites $\Delta \mathrm{p}_{\pi}$ and $\Delta \mathrm{p}_{\mu}$, and to the square of the transverse angle $\theta_{\pi}$ accepted of the $\pi$-mesons, according to

$\mathrm{dn} / \mathrm{dt}=\alpha\left(\mathrm{p}_{0}, \mathrm{p}_{\pi}, \mathrm{p}_{\mu}, \theta_{\pi}\right) \mathrm{I}_{\mathrm{p}} \Delta \mathrm{p}_{\pi} \Delta \mathrm{p}_{\mu} \theta_{\pi}^{2}$

(1)

where $\alpha\left(\mathrm{p}_{0}, \mathrm{p}_{\pi}, \mathrm{p}_{\mu}, \theta_{\pi}\right)$ is a constant that depends on the momentum $\mathrm{p}_{0}$ of the primary protons, on the central momentum values $\mathrm{p}_{\pi}$ and $\mathrm{p}_{\mu}$ of the collected $\pi$ and $\mu$ mesons, and on the angle $\theta_{\pi}$ of the production direction of the $\pi^{ \pm}$mesons. The constant can be estimated by inspection of Figures 2 and 3. According to Eq. (1), in order to obtain the same rate of production one can trade momentum spreads and angular acceptance with proton beam intensity. As an example, proton-beam intensity by a factor 150 higher than that of the Collaboration Proposal [1] (that is $150 \mathrm{MW}$ versus $1 \mathrm{MW}$ ) yields the same production rate with a momentum bite 10 times smaller and an angular spread also 4 times smaller. At the same time, as it is possible to see from Figures 2 and 3, by accepting a smaller angle, one optimizes the production, with a proper choice of the 
central momenta, by eliminating the need to collect particles at the edge of the distribution where the collection is less efficient and makes the beam excessively too large and diluted.

\section{The Proton Driver}

The Proton Driver (PD) is sketched in Figure 5. It is made of a 2-GeV proton SuperConducting Linac (pSCL) followed by a re-circulator where the proton beam makes four passes through 3 segments of SCL each for an energy gain of $1 \mathrm{GeV}$. The final energy is $15 \mathrm{GeV}$ and the average beam intensity is $10 \mathrm{~mA}$ (positive ions). The average proton power in exit is thus $150 \mathrm{MW}$. The proton beam power is essentially determined by the final energy, whereas the beam intensity is relatively modest and well within present technology. Moreover the Proton Driver is operating in a Continuous Wave mode and the proton beam is bunched at $200 \mathrm{MHz}$, that is essentially continuous. The 2-GeV SCL injector has a front-end operating at $201.25 \mathrm{MHz}$, followed by an intermediate section at $805 \mathrm{MHz}$, and a final section at $1.61 \mathrm{GHz}$. Thus the beam is bunched at $200 \mathrm{MHz}$ and is made of one bunch every eight $\mathrm{rf}$ buckets in exit of the injector Linac. The three sectors that make the re-circulator are all operating at $1.61 \mathrm{GHz}$. The main parameters of the Proton Driver are given in Table 1. In particular, the beam emittance, dimension, and momentum spread in exit are very small. Because the beam intensity in the Linac is relatively low, no space-charge effects are significantly expected. The concern with the possible formation of a beam halo is removed as well as the consequences on activation caused by uncontrolled beam losses. At the end of the traversal of each Linac section of the re-circulator the beam velocity is always very close to the speed of light, and the penalty on the acceleration rate from the transit-time factor by keeping the cavity cell length fixed, adjusted to exactly a half of the rf wavelength, is minimal.

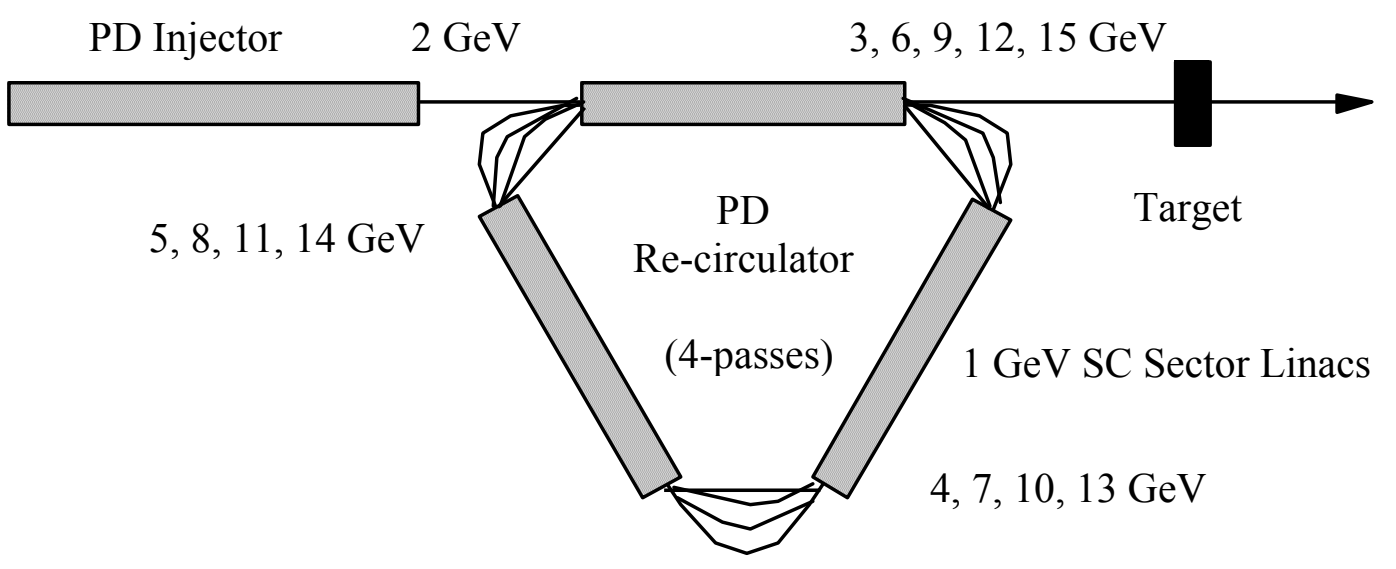

Figure 5. 15-GeV, Proton Driver (150-MW, 10-mA CW) 
Table 1. Proton Driver Parameters

\begin{tabular}{|l|l|}
\hline Mode of Operation & Continuous Wave (CW) \\
\hline Final Kinetic Energy & $15 \mathrm{GeV}$ \\
\hline Average Proton Current & $10 \mathrm{~mA}$ \\
\hline Average Proton Power & $150 \mathrm{MW}$ \\
\hline SCL-Injector Energy & $2 \mathrm{GeV}$ \\
\hline SCL- Injector Frequency & $201.25 \mathrm{MHz}$ to $116 \mathrm{MeV}$ \\
& $805 \mathrm{MHz}$ to $400 \mathrm{MeV}$ \\
& $1.61 \mathrm{GHz}$ to $2 \mathrm{GeV}$ \\
\hline Number of Passes in SCL-Sectors & $4(5 \mathrm{in}$ the first Linac Sector) \\
\hline Energy Gain for each Pass & $1 \mathrm{GeV}$ \\
\hline Frequency of SCL-Sectors & $1.61 \mathrm{GHz}$ \\
\hline Extracted Beam Emittance & $0.3 \pi \mathrm{mm}-\mathrm{mrad}$ (rms, norm.) \\
\hline Extracted Bunch Area & $1.0 \pi \mathrm{MeV}$-degree at $1.61 \mathrm{GHz}$ \\
\hline rms Momentum Spread & $<1 \mathrm{x} 10^{-4}$ \\
\hline rms Bunch Length & $<1 \mathrm{~mm}$ \\
\hline Beam Bunching Frequency & $201.25 \mathrm{MHz}, 1$ bunch every $8 \mathrm{rf}$ buckets \\
& at $1.61 \mathrm{GHz}$ \\
\hline Bunch Spacing & $150 \mathrm{~cm}$ \\
\hline
\end{tabular}

\section{Targetry}

The proton beam hits a solid target, for instance made of gold or tungsten; a liquid mercury target is not required for this scheme. The proton beam in exit of the Driver is already bunched at $200 \mathrm{MHz}$ with a spacing between bunches of about $150 \mathrm{~cm}$. The proton bunches have very small length and momentum spread. Also the beam transverse cross-section is small. Thus, the beam does not need to be manipulated further. Since otherwise the beam is continuous, no thermal shock effects on the target are expected. At the turn-on of the operation the beam intensity will be gradually raised at a tolerable rate without creating thermal stress. It is indeed a fact that, as it was determined from studies done for the Accelerator Production of Tritium (APT) project [4], a solid target can absorb considerably more beam power in the continuous mode of operation than in pulsed mode at low duty cycle.

Yet, even in the continuous mode of operation, there is a limit to the energy deposition on the target that we take here to be $150 \mathrm{~kW} / \mathrm{cm}^{2}$. A solution that allows spreading the proton beam over the target, and, at the same time, re-constructing the small cross-section of the $\pi$-meson beam is shown in Figure 6. Two dipole magnets B1 and B2 are located at the upstream side of the target separated by about $10 \mathrm{~m}$. Both magnets have the length of about one meter and capable of an excitation of $\pm 7.5 \mathrm{kG}$. The magnet power supply is oscillating at the frequency of $1 \mathrm{MHz}$, so that the beam entering the system is swept across the cross-section of the target over a squared area of $33 \times 33 \mathrm{~cm}^{2}$. The sweeping actually occurs both horizontally and vertically. At this purpose the magnet system is duplicated with another pair (not shown in the Figure 6) oriented perpendicular to each other. Assuming that the target length is $30 \mathrm{~cm}$, the target traversal time by the beam is about one nanosecond, and the total target area is swept in about one microsecond so that 
any region of the target does not receive more than $0.1 \%$ of the total beam power, that is $150 \mathrm{~kW} / \mathrm{cm}^{2}$. Beforehand, though, and simultaneously, the beam is focused on the target to an amplitude value $\beta^{*}=150 \mathrm{~m}$, that yields locally a beam spot size of $1.7 \mathrm{~mm}$ ( $\mathrm{rms}$ value). The same operation, but with the sequence inverted, is done on the other side of the target, the receiving end of the $\pi^{ \pm}$mesons coming out of the target. A similar sweeping system of magnets, B3 and B4, are located at the downstream end of the target. The beam in exit is counter-swept at the same frequency of $1 \mathrm{MHZ}$ and the beam image re-constructed at the exit of the B4 magnet as shown in Figure 6. At the exit of the B4 sweeping magnets the $\pi^{ \pm}$mesons enter the solenoid magnet. Because the $\pi^{ \pm}$mesons have a considerably lower momentum than the primary protons, it will be easier to bend them, and the second half of the bending system, to the right of the target, is considerably easier to conceive, design and build. The magnet system at the entrance, of course, represents a more challenging technical difficulty; but it should be possible to build magnets operating with an oscillating power supply at the $1-\mathrm{MHz}$ frequency. Of course, other solution may be possible and can be proposed.

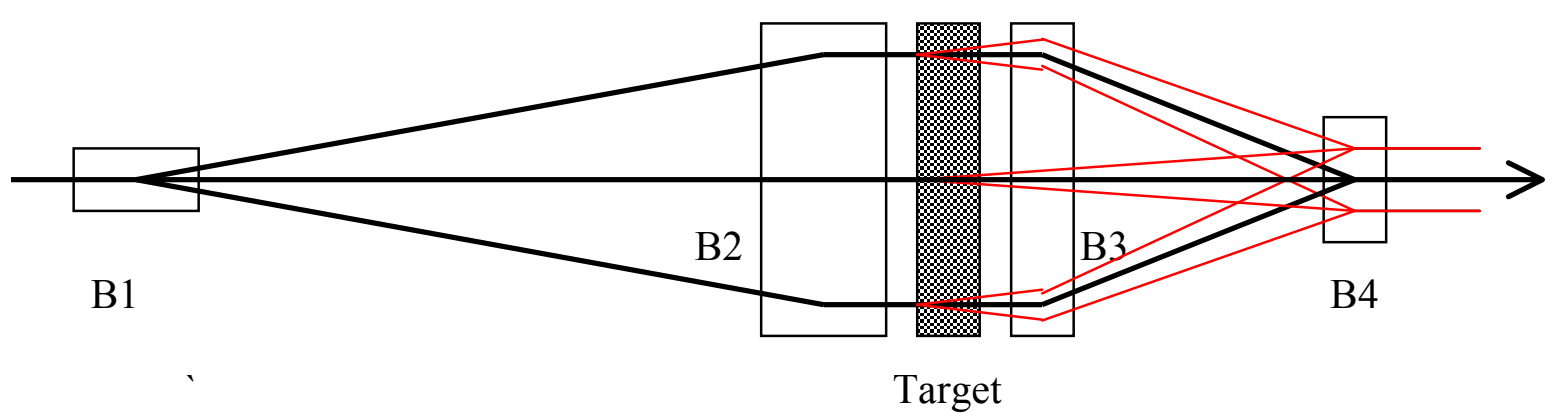

Figure 6 . Targetry of the protons and refocusing of the $\pi^{ \pm}$mesons with sweeping magnets

\section{The $\pi$ - $\mu$ Production Channel}

We shall denote with $\mathrm{d}^{2} \sigma_{\pi} / \mathrm{dp} \pi \mathrm{d} \Omega$ the differential cross-section for the production of the $\pi^{ \pm}$- mesons. An inspection of Figures 2 and 3 shows that $\mathrm{d}^{2} \sigma_{\pi} / \mathrm{dp}_{\pi} \mathrm{d} \Omega \sim 1.5$ barn $/(\mathrm{GeV} / \mathrm{c})$ for both signs, around the central momentum value $\mathrm{p}_{\pi} \sim 600 \mathrm{MeV} / \mathrm{c}$, and at production angles $\theta_{\pi}$ having $0.973<\cos \theta_{\pi}<1$; that is $0<\theta_{\pi}<0.233 \mathrm{rad}$. We shall accept all the particle momenta in the range $500<\mathrm{p}_{\pi}<700 \mathrm{MeV} / \mathrm{c}$. The resulting total cross-section is then $\sigma_{\pi}=0.051$ barn. The production rate of the $\pi^{ \pm}$mesons of both sign is given by

$\mathrm{dn}_{\pi} / \mathrm{dt}=2 \sigma_{\pi} \ell \rho \mathrm{I}_{\mathrm{p}} / \mathrm{e}$

where $\ell \sim 30 \mathrm{~cm}$ is the target length, $\mathrm{I}_{\mathrm{p}} / \mathrm{e}=6.25 \times 10^{16} / \mathrm{s}$ is the proton intensity, and $\rho \sim 6 \times 10^{22}$ nucleus $/ \mathrm{cm}^{3}$ is the density of the target material (Au). The total production rate of the $\pi^{ \pm}$mesons is thus $\mathrm{dn}_{\pi} / \mathrm{dt}=1.12 \times 10^{16} / \mathrm{s}$, that is an yield of about $0.18 \pi / \mathrm{p}$.

The target is located inside a super-conducting solenoid magnet with a field $\mathrm{B}=20$ Tesla that will trap and transport the $\pi^{ \pm}$- mesons produced. Inside the magnet the mesons 
performs helical trajectories with a transverse radius $\mathrm{a}_{\pi}$ directly related to the production angle $\theta_{\pi}$ according to the relation

$\mathrm{a}_{\pi}=0.3 \mathrm{p}_{\pi} \theta_{\pi} / \mathrm{B}$

with $\mathrm{a}_{\pi}$ in $\mathrm{mm}, \mathrm{B}$ in Tesla, $\mathrm{p}_{\pi}$ in GeV/c, and $\theta_{\pi}$ in mrad. With our parameters the radius $\mathrm{a}_{\pi}$ varies between 1.75 and $2.45 \mathrm{~mm}$. The period of the helical trajectories is $63 \mathrm{~cm}$ for the central momentum. Nevertheless, because of their initial divergence, the $\pi$-meson beam in exit of the target has a large transverse dimension equal to a full width of $7 \mathrm{~cm}$ with a $30 \mathrm{~cm}$ long target, larger than the original proton spot size. The size of the $\pi^{ \pm}$- meson beam is thus still reasonably small, and can be accommodated in a sufficiently small magnet aperture. To avoid activation and unnecessary beam loss, the internal magnet diameter can be set to $30 \mathrm{~cm}$. Of course, inspection of Eq. (3) shows that there is a large range of values where to trade one parameter with respect to the others. The total emittance of the captured $\pi^{ \pm}$- meson beam is estimated to be $\varepsilon_{\pi}=16 \pi \mathrm{m}$-rad in exit of the target. To this one should add the contribution of the multiple Coulomb scattering of the electrically charged mesons in the target, that raise the total expected emittance of the $\pi$-meson beam to $\varepsilon_{\pi}=23 \pi \mathrm{m}$-rad.

The decay length of the $\pi$-mesons into $\mu$-mesons varies between 28 and $40 \mathrm{~m}$. We adopt the length of $35 \mathrm{~m}$ for the transport in the solenoid magnet. Thus about $63 \%$ of the $\pi^{ \pm}$ mesons will have had a chance to decay. At the moment of production the $\pi^{ \pm}$- meson beam has the same longitudinal distribution of the beam of the primary protons. At end of the transport, each bunch is tilted because of the velocity difference between the top and the bottom of the momentum distribution. This will cause a total final extension of each bunch of $63 \mathrm{~cm}$, less than half of the bunch spacing. The momentum spread still ranges between 500 and $700 \mathrm{MeV} / \mathrm{c}$. The parameters of the $\pi$-meson production are summarized in Table 2.

Table 2. Parameters of Production of the $\pi$ Mesons

\begin{tabular}{|l|l|}
\hline Target Material & Gold $(\mathrm{Au})$ \\
\hline Target Length & $30 \mathrm{~cm}$ \\
\hline Central Momentum & $600 \mathrm{MeV} / \mathrm{c}$ \\
\hline Momentum Range & $500-700 \mathrm{MeV} / \mathrm{c}$ \\
\hline Angular Acceptance & $0.233 \mathrm{rad}$ \\
\hline Differential Cross-Section & $1,500 \mathrm{mb} /(\mathrm{GeV} / \mathrm{c})$ \\
\hline Total Cross-Section & $51 \mathrm{mb}$ \\
\hline Production Rate & $1.12 \times 10^{16} \pi / \mathrm{s}$ \\
\hline Yield & $0.18 \pi / \mathrm{proton}$ \\
\hline Solenoid Field & $20 \mathrm{Tesla}$ \\
\hline Length of Production Channel & $35 \mathrm{~m}$ \\
\hline Beam cross-section Radius & $3.5 \mathrm{~cm}$ \\
\hline Total Emittance & $23 \pi \mathrm{m}-\mathrm{rad}$ \\
\hline Bunch Spacing & $150 \mathrm{~cm}$ \\
\hline Final Long. Extension & $\pm 31 \mathrm{~cm}$ \\
\hline
\end{tabular}




\section{$\mu$-Production}

Each $\pi$ meson decays in a $\mu$ meson essentially directed forward. The momentum distribution of the $\mu^{ \pm}$mesons is about uniform in the range from 0.6 and 1.0 of the $\pi^{ \pm}$ mesons momentum value. We shall accept $\mu^{ \pm}$mesons with a central value of $450 \mathrm{MeV} / \mathrm{c}$ and a full momentum bite of $22.5 \mathrm{MeV} / \mathrm{c}(5 \%)$. The distribution of the $\mu^{ \pm}$mesons in the transverse phase space follows closely the "footprint" of the $\pi^{ \pm}$mesons with a normalized (full) emittance of $27 \pi \mathrm{m}$-rad in both planes. To this we should also add the contribution from multiple Coulomb scattering that raises the total final $\mu$-meson beam emittance to $37 \pi \mathrm{m}$-radThe longitudinal distribution preserves the original bunching at $200 \mathrm{MHz}$, and each $\mu$-bunch has the same total extension $(63 \mathrm{~cm})$ of the $\pi$-bunches at the moment of their decay. The full bunch area is thus expected to be about $0.024 \pi \mathrm{eV}-\mathrm{s}$, with an energy spread of $\pm 10 \mathrm{MeV}$. The $\mu$ production rate is finally $0.88 \times 10^{15} \mu / \mathrm{s}$, that is a yield of $0.014 \mu / \mathrm{p}$. The yield is about a factor 40 lower than that of the corresponding design of the Collaboration Proposal [1]; but since the proton beam intensity is a factor 150 times larger, actually the overall $\mu$ production rate is about a factor 4 higher. At the same time one has avoided, with the present scheme, difficult bunch manipulation and rotation, and ionization cooling. During production in the transport channel, the maximum Larmor radius of the $\mu$-beam cross-section is $5.6 \mathrm{~mm}$, but, like the original $\pi$-meson beam, also the $\mu$-mesons spread over a full width of $7 \mathrm{~cm}$. Thus it fits well within the available physical aperture of the solenoid magnet. The main parameters of the $\mu$-production are summarized in Table 3.

Table 3. Parameters of Production of the $\mu$ Mesons

\begin{tabular}{|l|l|}
\hline Central Momentum & $450 \mathrm{MeV}$ \\
\hline Momentum Bite (full) & $22.5 \mathrm{MeV}$ \\
\hline Momentum Spread (full) & $5 \%$ \\
\hline Energy Spread & $\pm 10 \mathrm{MeV}$ \\
\hline Production Rate & $0.88 \times 10^{15} \mu / \mathrm{s}$ \\
\hline Yield & $0.014 \mu /$ proton \\
\hline$\mu$-Beam cross-section Radius & $3.5 \mathrm{~cm}$ \\
\hline Norm. Emittance (full) & $37 \pi \mathrm{m}-\mathrm{rad}$ \\
\hline Bunch Separation & $150 \mathrm{~cm}$ \\
\hline Bunching Frequency & $201.25 \mathrm{MHz}$ \\
\hline Bunch Final Extension & $\pm 31 \mathrm{~cm}$ \\
\hline Bunch Area (full) & $0.024 \pi \mathrm{eV}-\mathrm{s}$ \\
\hline
\end{tabular}

\section{$\mu$-Acceleration}

The interesting feature of the scheme proposed here for the Neutrino Factory is that the $\mu$-beam at the end of the transport in the solenoid magnet has dimensions and spreads small enough that it can immediately be injected in a Super-Conducting Linac ( $\mu$ SCL). The Linac starts with a frequency of $200.125 \mathrm{MHz}$ to capture the beam bunches when they have large longitudinal spreads as at the moment of production. As during acceleration the bunches are shortened, the rf is then switched to $805 \mathrm{MHz}$ for a more efficient acceleration. Also, during the first section of the $\mu \mathrm{SCL}$, rf cavities are separated 
by super-conducting solenoid magnets to provide focusing and the transverse confinement of the beam. In later accelerating sections, after the beam transverse emittance has been sufficiently reduced by adiabatic damping, the solenoid magnets can be replaced with ordinary quadrupole magnets. There is no need of bunch rotation or other rf manipulation before final acceleration. There is also no need of ionization cooling since the beam dimensions are already satisfactorily small. Eventually, if necessary, because of the relatively large transverse dimension, the first section of the accelerating device can be an Induction Linac that provides larger transverse aperture and can simultaneously add acceleration and transverse focusing. This then will be followed by a SCL proper. Actually, it may also be possible that the entire acceleration can take place in a SCL from the start; a solution that we mostly prefer. The final energy is 32 $\mathrm{GeV}$ when the momentum spread is less than $1 \%$, the bunch length of few centimeters, and the actual full betatron emittance $122 \pi \mathrm{mm}-\mathrm{mrad}$.

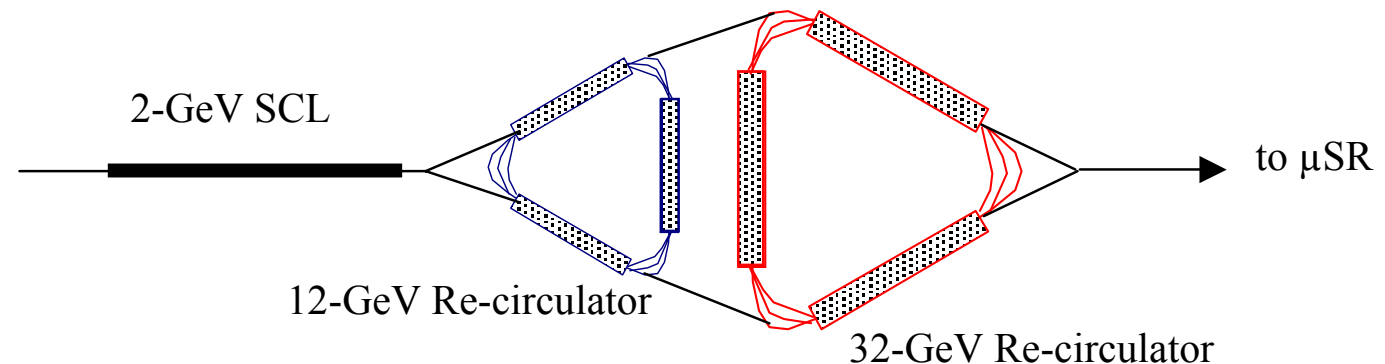

Figure 7. $\mu$-Acceleration to $32 \mathrm{GeV}$

Table 4. Parameters of the Muon SC Linacs

\begin{tabular}{|l|l|}
\hline Mode of Operation & Continuous Wave (CW) \\
\hline Final Kinetic Energy & $32 \mathrm{GeV}$ \\
\hline Average Muon Current & $2 \times 0.14 \mathrm{~mA}$ \\
\hline Average Muon Power & $2 \times 4.5 \mathrm{MW}$ \\
\hline SCL-Injector Energy & $2 \mathrm{GeV}$ \\
\hline SCL- Injector Frequency & $201.25 \mathrm{MHz}$ to $800 \mathrm{MeV}$ \\
& $805 \mathrm{MHz}$ to $2 \mathrm{GeV}$ \\
\hline Energy of First Re-Circulator & $12 \mathrm{GeV}$ \\
\hline Energy of Second Re-Circulator & $32 \mathrm{GeV}$ \\
\hline Number of Passes in SCL-Sectors & $3\left(4 \mathrm{in}\right.$ the $1^{\text {st }}$ Sector of each Re-Circulator) \\
\hline Energy Gain for each Pass & $1 \mathrm{GeV}$ for $1^{\text {st }}$ Re-Circulator \\
& $2 \mathrm{GeV}$ for $2^{\text {nd }}$ Re-Circulator \\
\hline Frequency of Re-Circulator Linacs & $1.61 \mathrm{GHz}$ \\
\hline Extracted Beam Emittance & $122 \pi \mathrm{mm}-\mathrm{mrad}$ (full) \\
\hline Extracted Bunch Area & $0.024 \pi \mathrm{eV}-\mathrm{s}$ \\
\hline Momentum Spread & $\pm 3 \times 10^{-3}$ \\
\hline Bunch Length & $\pm 0.2 \mathrm{~ns}$ \\
\hline Beam Bunching Frequency & $201.25 \mathrm{MHz}$ \\
\hline Bunch Spacing & $150 \mathrm{~cm}$ \\
\hline
\end{tabular}


The layout of the $\mu \mathrm{SCL}$ is sketched in Figure 7 and follows closely the design of the Collaboration Proposal [1]. There are three parts: (1) a 2-GeV SCL, followed by (2) a three-sided re-circulator for acceleration to $12 \mathrm{GeV}$, and (3) a larger final re-circulator, also three-sided, for acceleration to $32 \mathrm{GeV}$. Each of the three sides of the re-circulators are made of SCL, and at each pass the energy gain is about $1 \mathrm{GeV}$ in the first and $2 \mathrm{GeV}$ in the second re-circulator. The number of beam passes in each re-circulator is thus 3 . The rf in both re-circulators is always $805 \mathrm{MHz}$. The main parameters of the muon Super-Conducting Accelerators to $32 \mathrm{GeV}$ are shown in Table 4.

\section{$\mu$-Storage and Decay in Neutrinos}

At the end of the acceleration, the muons, that have now a lifetime of $0.67 \mathrm{~ms}$, are first separated according to their charge and then injected into a storage ring in opposite long straights as shown in Figure 8. In the $\mu$ SR the muons of opposite sign circulate in opposite directions waiting for their decay into a pair of neutrinos. A full beam divergence of $0.067 \mathrm{mrad}$ is required so that the spot size at the distance of about 3,000 $\mathrm{km}$ does not exceed $\pm 100 \mathrm{~m}$. This requires a final focus of the $\mu$-beam with $\beta^{*} \sim 110 \mathrm{~km}$. A single high-beta insertion is provided in each of the two long straight sections of the muon storage ring from where the neutrinos escape toward the desired location. Otherwise the design of the $\mu$ SR follows very closely that of the Collaboration Proposal [1]. The storage ring has a periodicity 2 . There are two arcs separated by two 500-m long straight sections. In each arc there are eight super-conducting bending magnets with a bending field of 8 Tesla. The ring circumference is about 1.1 kilometer, the transverse width is $35 \mathrm{~m}$, and the shape is elongated to allow that most of the neutrinos $(\sim 45 \%)$ escape along the desired direction from each of the long straight-sections. To preserve during the storage the initial beam bunching and longitudinal shape, it may be necessary to install a $201.25 \mathrm{MHz}$ (or higher) rf cavity system. The main parameters of the $32-\mathrm{GeV}$ muon Storage Ring are summarized in Table 5.

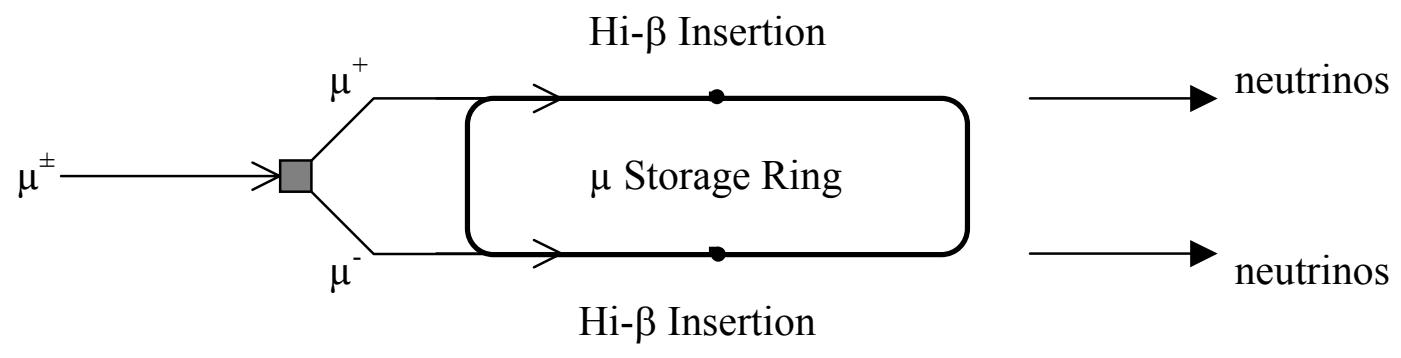

Figure 8. Injection and Circulation of the $\mu^{ \pm}$in the $32-\mathrm{GeV}$ Storage Ring

\section{The $\mu$-Collider}

The Neutrino Factory we have described can also be used as the muon source to inject into a high-luminosity high-energy $\mu^{+}-\mu^{-}$Collider. The beam of muons of both sign, at the exit of the $32-\mathrm{GeV} \mu \mathrm{SCL}$, by-passes the $\mu \mathrm{SR}$, and is accelerated even further to a considerable higher energy that we take here to be $1 \mathrm{TeV}$ for each charge species. The 
two charges are then separated, individually transported, and injected into one-ring collider proper. The outline of the process is sketched in Figure 9.

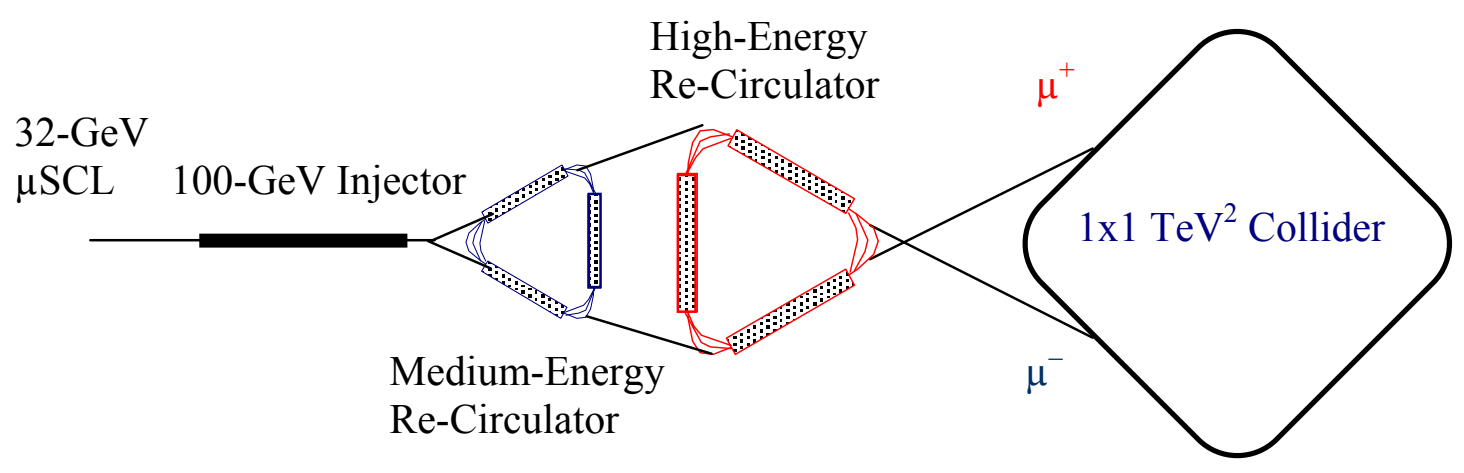

Figure 9. High-Energy SCL Accelerators and $1 \mathrm{x} 1 \mathrm{TeV}^{2} \mu^{+}-\mu^{-}$Collider

Table 5. Parameters of the Muon Storage Ring

\begin{tabular}{|l|l|}
\hline Kinetic Energy & $32 \mathrm{GeV}$ \\
\hline Magnetic Rigidity & $107.026 \mathrm{~T}-\mathrm{m}$ \\
\hline$\beta$ & 0.999994585 \\
\hline$\gamma$ & 303.86 \\
\hline Momentum & $32.105 \mathrm{GeV} / \mathrm{c}$ \\
\hline Circumference & $1112.077 \mathrm{~m}$ \\
\hline Revolution Frequency $\quad 0.2696 \mathrm{MHz}$ \\
\multicolumn{1}{|c|}{ Period } & $3.7095 \mu \mathrm{s}$ \\
\hline Periodicity & $2 \mathrm{w} / \mathrm{mirror}$ symmetry \\
\hline Total Number of Dipole Magnets & 16 \\
\hline Dipole Field & $8 \mathrm{Tesla}$ \\
\hline Magnet Length & $5.254 \mathrm{~m}$ \\
\hline Bending Radius & $13.378 \mathrm{~m}$ \\
\hline Packing Factor in the Arcs & 0.75 \\
\hline Length of an Arc & $56.04 \mathrm{~m}$ \\
\hline Width of the Storage Ring & $35.675 \mathrm{~m}$ \\
\hline Length of the Insertions & $500 \mathrm{~m}$ \\
\hline Number of Hi-Beta Insertions & $1 \mathrm{per} \mathrm{Long} \mathrm{Straight}$ \\
\hline Hi-Beta value & $110 \mathrm{~km}$ \\
\hline Beam Spot Radius at Hi-Beta location & $3.6 \mathrm{~m}$ \\
\hline Beam Divergence at Hi-Beta location & $\pm 0.0333 \mathrm{mrad}$ \\
\hline Muon Lifetime & $0.67 \mathrm{~ms}$ \\
\hline Total Number of muons stored & $5.9 \mathrm{x} 10^{11}$ \\
\hline Circulating Current & $25.4 \mathrm{~mA}$ \\
\hline & \\
\hline
\end{tabular}

The muon High-Energy Accelerator ( $\mu \mathrm{HEA}$ ), that accelerates both signs of muons from 32 to $1000 \mathrm{GeV}$, follows a layout similar to the $\mu \mathrm{SCL}$ described above that accelerates 
muon from 0.45 to $32 \mathrm{GeV}$, with the exception that now a high frequency rf cavity system (1.6-3.2 GHz) is used for more compact and more efficient acceleration (an average of $25 \mathrm{MeV} / \mathrm{m}$, as suggested by the TESLA project). The $\mu \mathrm{HEA}$ is made of a SC Linac for acceleration to $100 \mathrm{GeV}$, a Medium-Energy Re-Circulator that accelerates to $300 \mathrm{GeV}$, and a High-Energy Re-circulator for the final acceleration to $1 \mathrm{TeV}$. As in the $\mu \mathrm{SCL}$, also the $\mu \mathrm{HEA}$ accelerates simultaneously both signs of muons. The main parameters of the $\mu \mathrm{HEA}$ are given in Table 6 . Both Re-circulators are 3-sided, and the beam makes three passes in each linac section. In the first Re-Circulator the energy gain per SCL-section is $30 \mathrm{GeV}$, in the second $60 \mathrm{GeV}$. The average beam current and final power are relatively low. Most of the cost is for the manufacturing of the rf cavities and cryostats.

Table 6. Parameters of the Muon High-Energy Accelerator

\begin{tabular}{|l|l|}
\hline Mode of Operation & Continuous Wave $(\mathrm{CW})$ \\
\hline Final Kinetic Energy & $1 \mathrm{TeV}$ \\
\hline Average Muon Current & $2 \times 0.14 \mathrm{~mA}$ \\
\hline Average Muon Power & $2 \times 141 \mathrm{MW}$ \\
\hline SCL-Injector Energy & $100 \mathrm{GeV}$ \\
\hline SCL- Injector Frequency & 1.6 to $3.2 \mathrm{GHz}$ \\
\hline Energy of First Re-Circulator & $300 \mathrm{GeV}$ \\
\hline Energy of Second Re-Circulator & $1000 \mathrm{GeV}$ \\
\hline Number of Passes in SCL-Sectors & $3(4$ in the first Linac Sector of each \\
& Re-Circulator) \\
\hline Energy Gain for each Pass & $30 \mathrm{GeV}$ for $1^{\text {st }}$ Re-Circulator \\
& $60 \mathrm{GeV}$ for $2^{\text {nd }}$ Re-Circulator \\
\hline Frequency of Re-Circulator Linacs & $3.2 \mathrm{GHz}$ \\
\hline Extracted Beam Emittance & $3.9 \pi \mathrm{mm}-\mathrm{mrad}($ full) \\
\hline Extracted Bunch Area & $0.024 \pi \mathrm{MeV}$-degree at $1.61 \mathrm{GHz}$ \\
\hline Momentum Spread & $\pm 1.0 \times 10^{-3}$ \\
\hline Bunch Length & $\pm 0.02 \mathrm{~ns}$ \\
\hline Beam Bunching Frequency & $201.25 \mathrm{MHz}$ \\
\hline Bunch Spacing & $150 \mathrm{~cm}$ \\
\hline
\end{tabular}

The Collider has a circumference of $3.743 \mathrm{~km}$ and is made of 8-Tesla super-conducting bending magnets. The packing factor, that allows also insertions for two low-beta insertions, injection and $\mathrm{rf}$ cavities, is $70 \%$. The bending radius of the magnets is $417 \mathrm{~m}$. The ring has a periodicity of four, with internal mirror symmetry. There are four arcs separated by four straight insertions each about 100 meter long. The main parameters of the Collider are given in Table 7.

At $1 \mathrm{TeV}$ the muon lifetime is $20 \mathrm{~ms}$. Injection into the Collider is continuous at the rate of $8.8 \times 10^{14} \mu / \mathrm{s}$; thus the total number of muons circulating at any one time is $1.84 \times 10^{13}$ and constant. To get the highest value of luminosity possible one requires a small circumference of the storage ring, a fewer number of bunches with a lot of particles, and a small transverse cross-section of the beam. The best strategy is thus to stack successive beam turns in the momentum phase space and preserve the beam emittance to the original 
value at the source. We shall assume that a low-beta value of $\beta^{*}=10 \mathrm{~cm}$ at the crossing is achievable. Also the beam-beam crossing is in dispersion-less locations. The Collider capturing rf cavity system operates at $20 \mathrm{MHz}$, that is one tenth of the incoming bunching frequency. The beam is injected by performing "longitudinal painting" in the standing-by rf buckets where 10 beam bunches are deposited every turn. The total number of circulating bunches is thus 250 each with a total $7.35 \times 10^{10}$ muons. In order to reach the final intensity, initially a total of 1,670 turns are required. Injection and "longitudinal painting" continues at the same rate to compensate for the depletion of the circulating muons. Each turn is captured by the rf system and accelerated over to make room for the subsequent turns as in cyclotron or FFAG accelerator. With this mode of operation the expected (constant) luminosity is $0.9 \times 10^{31} \mathrm{~cm}^{-2} \mathrm{~s}^{-1}$.

Table 7. Parameters of the $\mu^{+}-\mu^{-}$Collider

\begin{tabular}{|l|l|}
\hline Collision Energy & $1 \mathrm{TeV}$ per beam \\
\hline Luminosity & $0.9 \times 10^{31} \mathrm{~cm}^{-2} \mathrm{~s}^{-1}$ \\
\hline Circumference & $3,743 \mathrm{~m}$ \\
\hline Periodicity & $4 \mathrm{w} / \mathrm{mirror}$ symmetry \\
\hline Length of Long Straights & $100 \mathrm{~m}$ \\
\hline Low-Beta $\beta^{*}$ & $10 \mathrm{~cm}$ (dispersionless) \\
\hline Muon Lifetime & $20.8 \mathrm{~ms}$ \\
\hline Total Muon stored & $1.84 \times 10^{13}$ \\
\hline rf Cavity System & $20.125 \mathrm{MHz}$ \\
\hline Number of Bunches & 250 \\
\hline Emittance, full & $3.9 \pi \mathrm{mm}-\mathrm{mrad}$ \\
\hline Revolution Frequency & $80.0 \mathrm{kHz}$ \\
\hline Period & $12.5 \mu \mathrm{s}$ \\
\hline Bending Field & $8.0 \mathrm{Tesla}$ \\
\hline Pending Radius & $417.0 \mathrm{~m}$ \\
\hline Number of injected turns / cycle & 0.70 \\
\hline Total Bunch Area & 1,670 \\
\hline Bunch Half Length & $393 \pi \mathrm{eV}-\mathrm{s}$ \\
\hline Total Beam Momentum Spread & $20 \mathrm{~ns}$ \\
\hline Circulating Current & $\pm 2 \%$ \\
\hline Method of Injection & $235 \mathrm{~mA}$ \\
\hline & Momentum Stacking \\
\hline
\end{tabular}

\section{References}

[1] Collaboration home page http://www.cap.bnl.gov/mumu/studyii

[2] M.J. Brennan, et al., "Upgrading the AGS to $1 \mathrm{MW}$ Proton Beam Power", presented to PAC 2001, Chicago, Illinois, June 2001.

[3] Harold Kirk, private communication. BNL, May 200.

[4] APT ${ }^{3} \mathrm{He}$ Target/Blanket Topical Report, Los Alamos National Laboratory report LA-12670-MS, (1993). 